\title{
E-fuels as chemical energy carrier under the aspects of costs and efficiency
}

Martin Rothbart, J. Rechberger

AVL List GmbH

This manuscript is not available according to publishing restriction. Thank you for your understanding. 